\title{
Performance Analysis of Emotion Classification Using Multimodal Fusion Technique
}

\author{
${ }^{1}$ Chettiyar Vani Vivekanand, \\ ${ }^{2}$ Department of ECE, RMK College of Engineering and Technology (Autonomous), \\ Puduvoyal, Tamilnadu, India.
}

${ }^{* *}$ Corresponding Author: vanivivekanand@rmkcet.ac.in

Received: 11.01.2021, Revised: 05.02 .2021 Accepted: 05.03.2021, Published: 16.04 .2021

DOI:

10.53409/mnaa/jesit/2103

Abstract: As the central processing unit of the human body, the human brain is in charge of several activities, including cognition, perception, emotion, attention, action, and memory. Emotions have a significant impact on human well-being in their life. Methodologies for accessing emotions of human could be essential for good user-machine interactions. Comprehending BCI (Brain-Computer Interface) strategies for identifying emotions can also help people connect with the world more naturally. Many approaches for identifying human emotions have been developed using signals of EEG for classifying happy, neutral, sad, and angry emotions, discovered to be effective. The emotions are elicited by various methods, including displaying participants visuals of happy and sad facial expressions, listening to emotionally linked music, visuals, and, sometimes, both of these. In this research, a multi-model fusion approach for emotion classification utilizing BCI and EEG data with various classifiers was proposed. The 10-20 electrode setup was used to gather the EEG data. The emotions were classified using the sentimental analysis technique based on user ratings. Simultaneously, Natural Language Processing (NLP) is implemented for increasing accuracy. This analysis classified the assessment parameters as happy, neutral, sad, and angry emotions. Based on these emotions, the proposed model's performance was assessed in terms of accuracy and overall accuracy. The proposed model has a 93.33 percent overall accuracy and increased performance in all emotions identified.

Keywords: Emotion Classification, EEG signals, Brain Computer Interface, Multimodal Fusion, NLP.

\section{INTRODUCTION}

umans are renowned for their T behavioural oscillations controlled by their mental condition dependent by their emotions. New technologies hunger to enable machines to comprehend humans totally, and the accomplishment of real user-machine communications will be useless if a person's emotions are not managed correctly. As a result, the need for robots to comprehend human emotions has grown increasingly essential in recent years. Brain is the CPU of the human body, accessing it required to perceive what a person feels and thinks. Data from the brain may be gathered in various methods, including MRI scans, EEG, ECoG, MEG, and various other signals containing brain information. Among these, electroencephalogram (EEG) signals offer a wealth of information about a person's emotional state that may be used to understand brain psychology better. It is more beneficial to evaluate emotions using EEG signals since they are voltage-dependent signals, and it is challenging to affect the electrical activity tactically. Hence, classification of emotions using EEG data may support enhancing the BCI system. This system has received several uses in recent days, including medical and healthcare treatment. However, they might also be utilized as an assistive device for individuals who require physical and mental support to engage with the actual world.

This research examines current approaches for filtering gathered signals and provides a closer examination of existent EEG signals classification algorithms for effectively detecting emotion. This research further examines the usage of EEGs in numerous operations, as well as modern advances in interpreting signal features. The objective of this work is to provide an effective method for classification of EEG by employing the multimodal strategy, which not just examines EEG signals for emotions classifications, further checks results generated by the NLP techniques to achieve more accurate performances. The certain aspects under consideration are that the temporal channels pair ( $\mathrm{T}_{7}$ and $\mathrm{T} 8$ ) and the higher-frequencies band 
produced a greater outcome than was not reliant on the time of collected data. Multiple emotions may be classified using the proposed technique based on EEG data from the brain. To collect a wide variety of signals, the EEG signals capturing device with a limited channels was employed. The system is divided into three steps that entail acquiring the offline signals and doing pre-processing, which includes eliminating artifacts and outliers. Then utilizing that data for training the classifiers on a variety of emotions. Next stage was testing, which was gathered in practical and used by the classifiers to determine the subject's emotions. The individuals were then requested to view an image or listen to songs and remark on it as words in third step. The information is processed by NLP, and emotions are evaluated concurrently with the analysis of the EEG data collected at the moment. Both outcomes were merged to provide superior results.

Section II of the article outlines the fundamental ideas of the brain-computer interface. The in-depth literature study of current classification algorithms and the different uses of EEG data was dealt with in detail in the session that followed. Section IV delves further into the various signal gathering techniques and the proposed system, followed by Section V's conclusion and future development.

\section{RELATED WORK}

The EEG signal was utilized in a variety of studies for determining people's mental states. These signals aid in the analysis of the emotions of autistic children, who are typically considered to exhibit social and behavioural impairments, difficulty with stereotyped actions, and hesitation in interacting with others. The robot fixed to the laser sensors components was utilized for identifying the child and determine the range among them. The EEG wireless device was put on the child's scalp, and these signals were collected and analyzed to allow for additional interaction. Two modes of interaction were developed: dog mode, in which the robot detects child and travels around them, and follower mode, in which the robot walks away, enabling child to follow them based on the child's activity, as determined by monitoring EEG data. EEG signals may also be utilized to forecast how video advertising will be rated [1]. The multimodal method was presented in [2] in which EEG data was recorded while the patient watching an online video. Simultaneously, the video's worldwide rating was computed using NLP. The random forest-based strategy was utilized to predict rating using EEG data, and when tested on 25 participants, this fused approach produced promising results.
According to [3] EEG signals may distinguish between happy and sad emotions produced by images. PSD was used to extract features, while SVM was utilized as a classifier. The average accuracy of 71.20 percent was achieved after determining that the temporal pairs of channels and the higher-frequency bands produced the best results, regardless of the length of the data recorded. A multimodal approach was utilized in [4] to compare brain (EEG) and other peripheral data in order to identify emotion. The device used a variety of inputs, including an EEG, temperature, galvanic skin resistance, blood pressures, and breathing, to determine a person's emotions. Emotions were evoked using images representing various emotions of valance-arousal. This approach was employed to assess, and it was discovered that EEG signal performed greater than other secondary signals, leading to the conclusion that they are the best feature for interpreting a person's emotion.

According to the findings of the study [5] a person's inner state may be detected utilizing the EEG waves. The proper positioning of electrodes and selection of features for the test needs a more in-depth comprehension of each feature. On a selfrecorded dataset, the experiment was carried out by comparing all of the characteristics using machine learning algorithms. It was discovered that characteristics chosen using multivariate techniques work better, and that the optimum site to put electrodes was above the parietal Centroparietal lobe. In article [6] for BCI system, a novel functional system design was presented to address communication barriers caused by a lack of shared vocabulary. A taxonomy was created, which together provided a foundation for the BCI model, and its efficacy was tested against various comparable systems and discovered as efficient.

The BCI has gained popularity because it allows unhealthy individuals to act like normal people in the world. The study [7] examined all of the research efforts made to develop BCI research for persons who are abnormal with supporting benefits. The standard EEG-based BCI model was implemented to help physically challenged or paralyzed persons to accomplish their daily duties. The BCI system has progressed over several phases, and at this point, they might be employed in a broader range of applications. The BCIs began to comprehend a person's emotional state. Access to brain activity might give valuable information about the user's emotional condition. Understanding the emotion pattern, as described in [8], might be applied in two ways. Comprehending the emotional signals pattern allows them to be recognized and eliminated from the actual data stream. As a result, regardless of the user's mood, the user's goal is satisfied appropriately. Another benefit of a person's emotion is that it allows them to interact with other machines in a more natural manner. 
The two alternative feature selection approaches for EEG data were proposed in [9], concluding the features typically employed for emotion detection. The implemented approaches include statistical features related to Power Spectral Density (PSD) and High Order Crossings (HOC), and the outputs were obtained. The PSD strategy obtained $70 \%$ while the HOC approach achieved 69.5 percent, proving that both techniques are equally effective for identifying features in EEG data. The signal generation standard was important to EEG signal researchers since noise-free signals were the main objectives. As a result, they limit face, ocular, and jaw movements and relied on maximum electrodes to capture EEG signal, which was not feasible in a practical setting. Experiments were carried out on severely infested, noisy data with fewer electrodes ranging from four to six and average accuracy of $33 \%$ for happy, $33 \%$ for fear, $38 \%$ for anger, and 37.5 percent for sad was obtained in paper [10].

The study of EEG signal classification garnered a lot of attention. The efficacy of classification approaches was investigated in [11] for a movie induction experiment in which individuals spontaneously evoked actual emotion conditions and the EEG dataset of 6 cases was collected. The results on the EEG dataset led to a conclusion, including that the power spectral feature was better and that a linear dynamic system-based feature smoothing approach increases accuracy. Subjectindependent feature reduction with manifold learning can be used to show the course of emotional changes. A real-time multimodal BCI data collection metadata approach was presented in [12], which used the capacity to manipulate alpha waves deliberately through eye movements. Using alpha brain waves, this technology may be utilized to control external equipment such as wheelchairs. The reaction created by eye movement (detects and regulates the amplitudes of brain's alpha wave) was evaluated, analyzed, and sent into smart controller device. The occipital lobes send signal which distinguish the magnitude of alpha signals produced by the various motions. Individuals were instructed to shut and open the eyes during the testing time. They were able to use it to control the restricted motions of a robot. Similarly, the other movements were attempted as well. The system was able to complete the activity with the aid of the alpha waves.

\section{PROPOSED METHODOLOGY}

Human-computer interaction is in high demand, and the desire to do it organically has become the most significant requirement. The expectation of people that systems should comprehend what they think rather than just giving commands created the foundation for the BCI technology, which must be built efficiently to meet the needs of the present world. As seen in Figure 1, the $\mathrm{BCI}$ is broadly characterized:

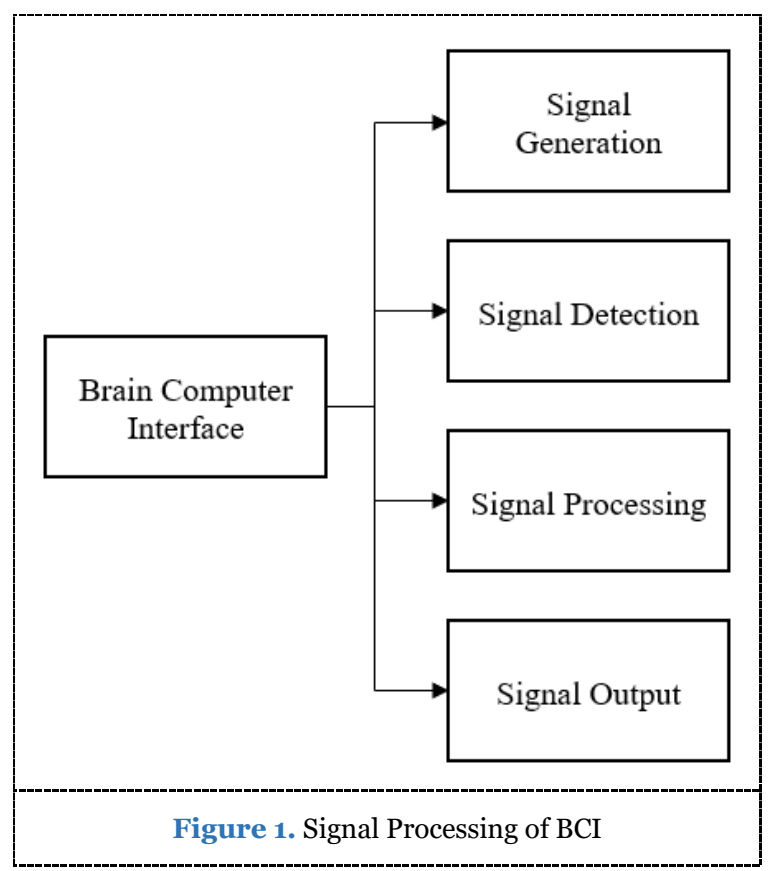

Signal Generation: The source of the signals must be a subject.

Signal Detection: The EEG kit's electrodes should identify them among other signals.

Signals Processing: The signal could contain noises and outliers, and it should be treated before they could be used.

Signal Output: It is necessary to obtain some meaningful information from processed signals.

Each of them is a large study field, and a more complete description is discussed below.

\subsection{Signal Generation}

The first stage is signal production, in which the subject (source of signal) must create signals, and there are two ways to do so. Collecting active signals via giving forced stimuli, exhibiting visuals, or asking people to envision motions is an essential technique to aid in real-time signal gathering. The alternative option is a passive approach in which the signals previously gathered from the patient are used. Actively producing signals is helpful because signal detection is easier when you have total control over the stimuli. However, it has the drawback that noises and outliers were very prevalent in active input, and electrode corrodes readily during practical trial use. Passively produced signal needs examining the whole subject's brain signals since the areas of interest are difficult to locate. 


\subsection{Signal Detection}

Different methods can be used to detect the brain signal. EEG signals are utilized to determine the brain's electrical activity, whereas fMRIs analyze the flow of blood in the brain. Aside from EEG and fMRI, there are additional signals such as MEG and ECoG. Each of them has its own benefits, thereby few rely on the temporal resolution and some special resolutions, like where and when it occurs.

\subsection{Signal Processing}

The primary issue with obtained EEG signal was that there is excessive noise in the data. Slight movements such as teeth grinding, eye and eyelid movements could result in a large variation in signal productions. The most essential task was to remove the noises, and then real signals may be utilized after this preprocessing. When the individual was actively participating in signals creation, the kind of signal and the moment of signal formation may be understood, making signal processing easier.

\subsection{Signal Transduction}

When the signal processing phase is completed, it indicates that the appropriate signals for analysis have been discovered. This signal must now be used efficiently to meet the subject's needs. The subject may have any purpose, such as moving a hand or legs, or, as in this example, it could just be aware of a person's feeling. The key point to keep in mind was that signals should be used effectively because they could not be gathered at any moment, and the whole system was inclined to error because the signals collected was too vulnerable.

The BCI is a direct communication channel that connects the brain to an external device. The objective of BCI is to assist, augment, or repair sensory-motor or cognitive functions, to develop applications for humans with disorders, to help visually impaired persons in visualizing external visuals, and to help paralyzed peoples in operating external systems without physical actions to decrypt the data stored on the brain. Human BCI EEG acquisition techniques are classified into three kinds. They are classified

- Invasive

- Partially invasive

- Non-invasive

During neurosurgery, the invasive kind involves placing electrodes exactly into the brain's grey matter. On the other hand, the partiallyinvasive approach places BCI device into the skull but keeps them externally on the brain. Because the electrodes are positioned outside the skull, the noninvasive technique is widely utilized in research. Electrocorticography (ECoG), which was a partly invasive method, and MRI and EEG, which were non-invasive and employ electrodes put on the head, are the most often used BCI techniques. EEG was utilized to monitor the brain's electrical activity, which was produced by nerve cells in billions known as neurons. This activity of EEG was monitored using electrodes attached to the head. EEG signals are classified into rhythmic frequencies varies from $0.5 \mathrm{~Hz}$ to $30 \mathrm{~Hz}$. As illustrated in Fig 2, EEG waves were classified as Alpha, Beta, Gamma, Theta and Delta. Subjects create brain signals (features) after adequate training, and the features of the stable EEG output that the person could consistently produce (waves) is utilized; one may further elicit the EEG signal with the external stimulation (induced potential).

Alpha has a high amplitude in the relaxed state, and beta has a high amplitude in the stimulated state. When a person transitions from a calm to an excited state, the amplitude change from alpha to beta waves may be clearly detected. To collect the EEG waves, a standard "10-20 System" with $10-20 \%$ spacing is utilized. Figure 2 depicts four types of lobes by using letters to represent the various areas of the brain's lobes.

- F - Frontal Lobe

- T - Temporal Lobe

- C - Central Lobe

- O- Occipital Lobe

Odd numbers to the left and even numbers to the right represent the exact position of electrode placements. The EEG is used to assess cognitive workload since it is less expensive, easier to use, provides wireless connectivity, and has a minimum cost maintenance. The EEG's raw data are typically contaminated with different artifacts, trends, and noises caused by eye blinks, muscle movements, and so forth.

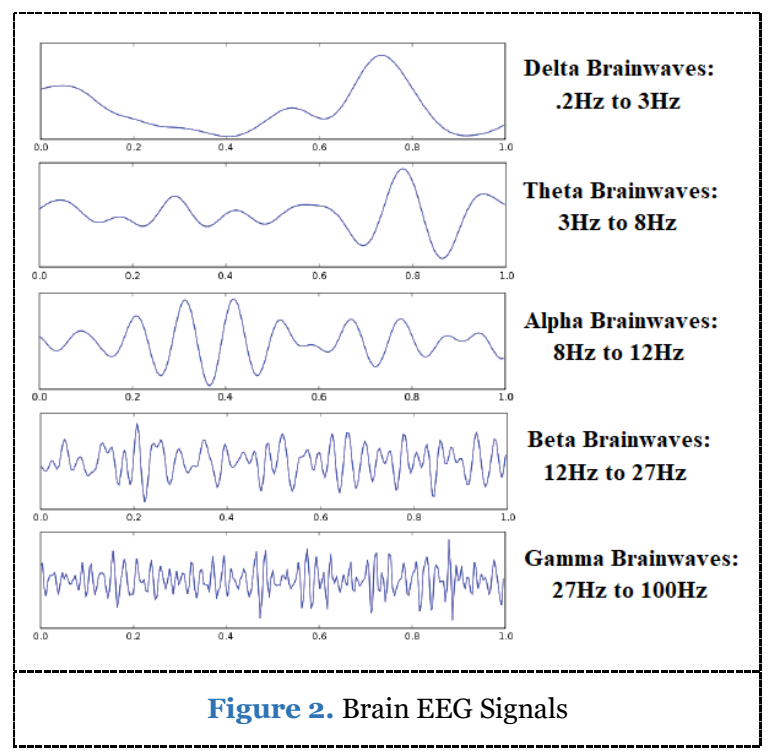


The proposed model classifies emotions by using EEG data, and its efficiency is tested using the NLP classification technique. The first stage is to perform two acts on the original EEG signals that were collected.

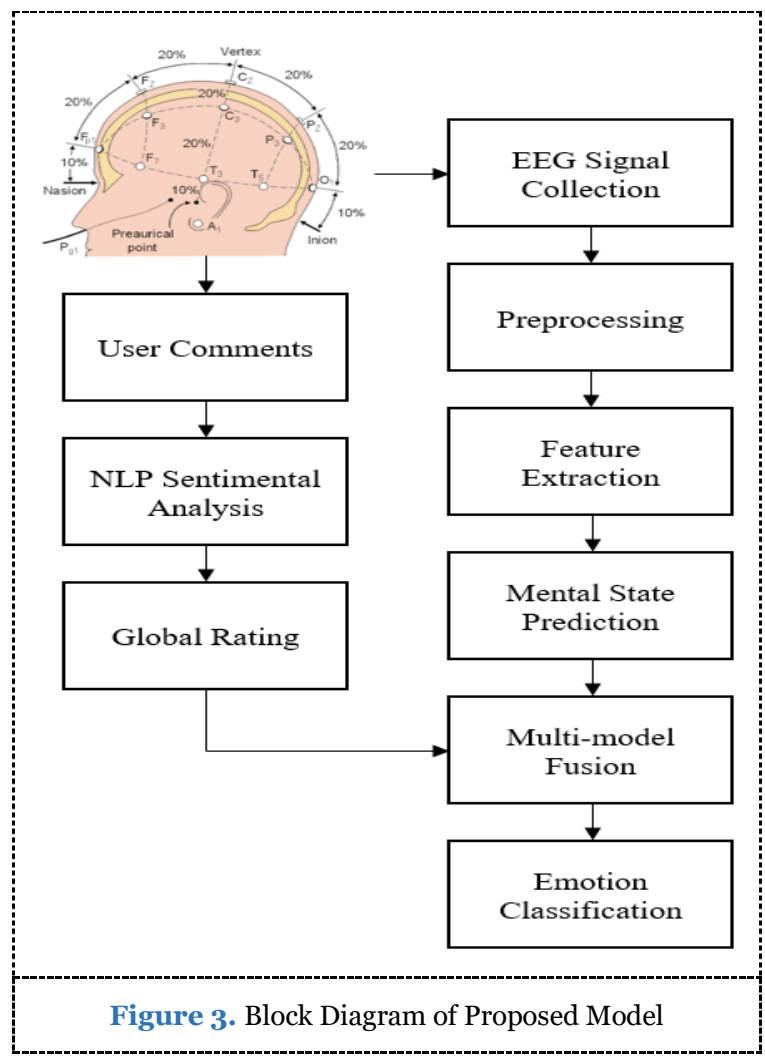

The first is independent component analysis, which breaks down multivariate arbitrary signals into collectively independent elements. Moving Average, in contrast was utilized for smoothing signals and changes every data value with surrounding average values. Before extracting features, the aforementioned approaches were employed to eliminate the influence of such undesirable artefacts. Later, random forest regression is used to do regression analysis on EEG data. The second stage is to use a natural language processing toolset to classify the subject's emotion based on their comments (text blob). Finally, to enhance the total predictions, the EEG-based rating was merged with an emotion score. Figure 3 depicts the whole planned model.

The proposed model was intended to classify four classes of emotions, every emotion has a count of subclasses, as seen in figure 4 . The basic emotions were easily classified using EEG signals, while the subclasses were only discovered with the use of NLP. As a result, the algorithm was able to efficiently classify emotions into one of the categories.

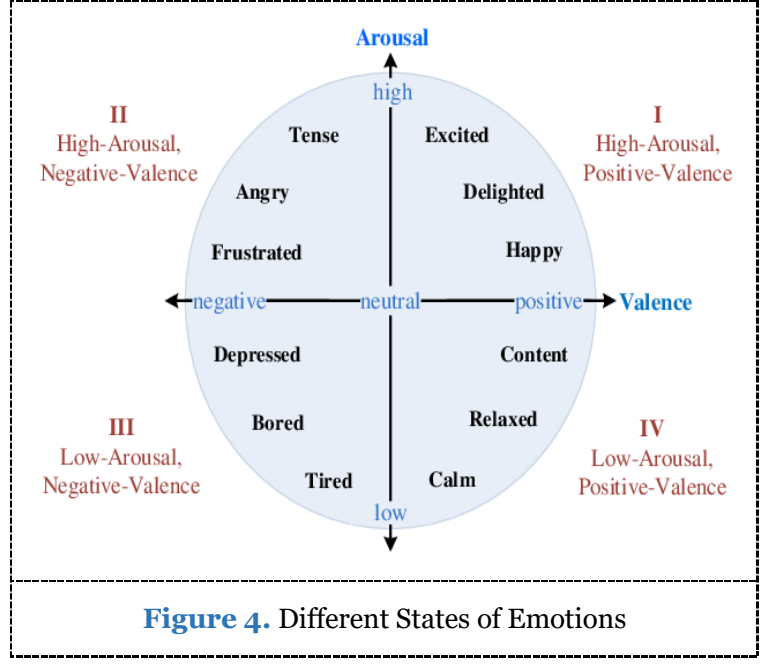

\section{EXPERIMENTAL RESULTS AND DISCUSSION}

In this section, the proposed model's performance is evaluated and compared with the existing techniques like ANN, Naïve Bayes, and SVM-DWT in terms of accuracy using different emotions like happy, neutral, angry, and sad. The proposed model is experimented with MATLAB Simulink tool. The performance analysis and the comparison of the proposed model is given in Table 1.

\begin{tabular}{cccccc}
\hline Models & Happy & Neutral & Sad & Anger & $\begin{array}{c}\text { Overall } \\
\text { Accuracy }\end{array}$ \\
\hline ANN & 89.24 & 89.92 & 88.34 & 91.16 & 89.66 \\
\hline NB & 91.36 & 90.55 & 89.75 & 90.68 & 90.58 \\
\hline $\begin{array}{c}\text { SVM- } \\
\text { DWT }\end{array}$ & 90.50 & 89.12 & 91.47 & 93.22 & 91.07 \\
\hline Proposed & 92.34 & 90.76 & 93.38 & 96.85 & 93.33 \\
\hline
\end{tabular}

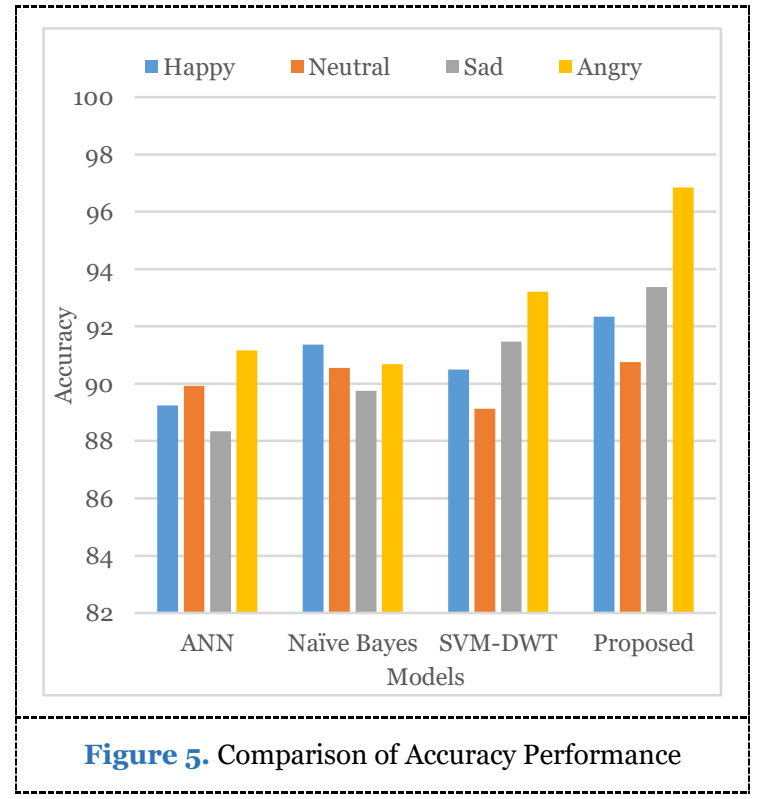




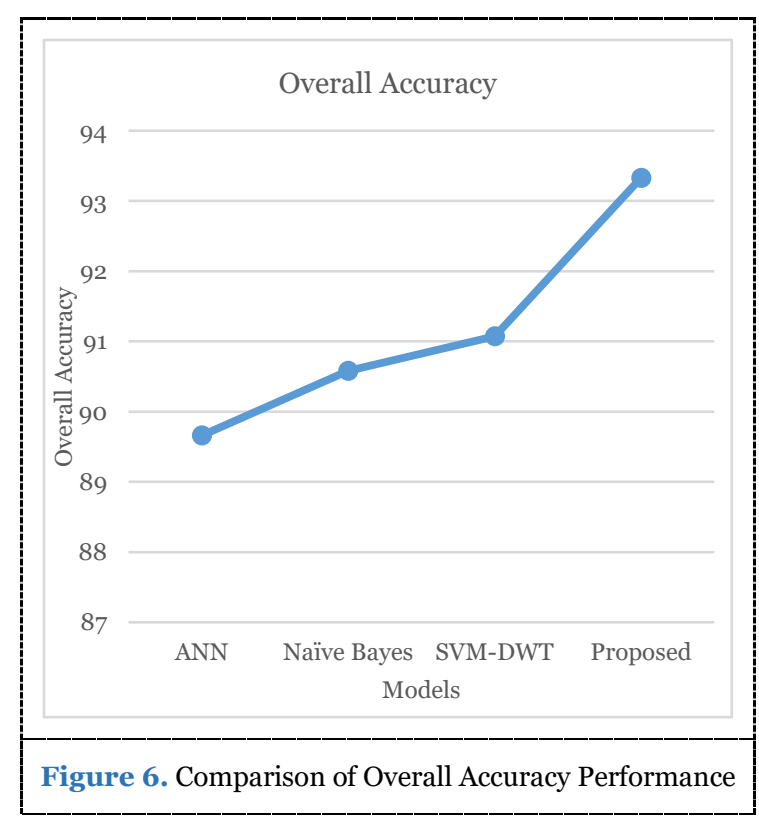

Figure 5 represents the comparison of the proposed model's accuracy performance of different emotions with the other existing techniques. Figure 6 represents the comparison of the proposed model's overall accuracy performance with the other existing techniques. The proposed model outperforms the other classifier models in terms of emotional state classification accuracy. Happy, Sad, Neutral, and Anger are the emotions classified and measured in the classification performance. The proposed model has an overall accuracy of $93.33 \%$ and improved performance in all the emotions classified. The overall accuracy difference between the proposed model and the other classifiers is $2.26 \%$ to $3.67 \%$.

\section{CONCLUSION AND FUTURE WORK}

Emotion identification is now provoking the attention of researches in a variety of areas, including those investigating the Brain-Computer Interface. This work proposed a multi-model fusion technique for emotion classification using BCI with EEG signals using different classifiers. The EEG signals were collected using the 10-20 electrode system. Using the sentimental analysis approach, the emotions were classified based on the user ratings. The emotions such as happy, neutral, sad, and anger were classified as the evaluation parameters in this work. The proposed model's performance was measured in terms of accuracy and overall accuracy based on these emotions. The proposed model has an overall accuracy of $93.33 \%$ and improved performance in all the emotions classified. The proposed model was compared with other models like ANN, Naïve Bayes, and SVMDWT. The overall accuracy difference between the proposed model and the different classifiers are between $2.26 \%$ to $3.67 \%$. There are several problems in hybrid BCI techniques, user-machine adaptation methods, and BCI reliability studies that take use of users' mental states. Future study anticipates that classification accuracy may be improved by extracting more significant characteristics from various domains such as frequency, time, or frequency domain.

\section{ETHICS APPROVAL AND CONSENT TO PARTICIPATE \\ Not applicable.}

\section{HUMAN AND ANIMAL RIGHTS}

No animals/humans were used for studies that are basis of this research.

\section{CONSENT FOR PUBLICATION \\ Not applicable.}

AVAILABILITY OF DATA AND MATERIALS

The authors confirm that the data supporting the findings of this research are available within the article.

\section{FUNDING}

None.

\section{CONFLICT OF INTEREST}

The authors declare no conflict of interest, financial or otherwise.

\section{ACKNOWLEDGEMENTS}

The authors would like to thank their present employer for providing support while carrying out this research work.

\section{REFERENCES}

[1]. Goulart C, Castillo J, Valadão C, Bastos T, Caldeira E. EEG analyses and mobile robots as tool for emotions characterizations in autisms. $5^{\text {th }}$ Congress of the Brazilian Biotechnology Society, 2013; pp. 1-2.

[2]. Gaubaa H, Kumar P, Roy PP, Singh P, Dogra DP, Raman B. Predictions of advertisements preferences by fusing EEG responses and sentiment analyses. Neural Netw. 2017; 92: 7788.

[3]. Jatupaiboon N, Panngum S, Israsena P. Real-Time EEGBased Happiness Detection System. Sci World J. 2013; 1(1): 1-12.

[4]. Khalili Z, Moradi M.H. Emotions Recognitions Systems Using Brain and Peripheral Signal using Correlations Dimensions to Improve the Result of EEGs. International Joint Conference on Neural Networks. 2009; pp. 1571-1575.

[5]. Robert J, Angelika P, Martin B. Features Extraction and Selections for Emotions Recognitions from EEGs. IEEE Trans Affective Comput. 2014; 5(3): 327-339.

[6]. Mason S G, Birch G.E. A General Frameworks for BrainComputer Interfaces Designs. IEEE Trans Neural Syst Rehabilitation Eng. 2003; 11(1): 70-85. 
[7]. Mandeep K, P. Ahmed, M. Q Rafiq. Technology Developments for Unblessed Peoples using BCI: A Survey. Int J Comput Appl. 2012; 40(1): 18-24.

[8]. Gary G M, Tsvetomira T, Anton N. Emotional BrainComputer Interface. Int J Auto Adaptive Communi Syst. 2013; 6(1): 9-25.

[9]. Bastos-Filho TF, Ferreira A, Atencio AC, Arjunan S, Kumar D. Evaluations of Features Extraction Technique in Emotional States Recognitions. International conference on intelligent human computer interaction. 2012; pp. 1-6.

[10]. Mina M, El-Ayat K, Coan JA, Allen JJ. Using minimal numbers of electrode for emotions detections using brain signal produced from a new elicitation technique. Int J Auto Adaptive Communi Syst. 2013; 6(1): 80-97.

[11]. Xiao-W W, Dan N, Bao-L L. Emotional states classifications from EEGs data using machines learning approach. Neurocomput. 2013; 129: 94-106.

[12]. Rohan Hundia. Brain-Computers Interfaces-Controlling Device utilizing the Alpha Brain Wave. Int J Sci Technol Res 2015; 4(1): 281-285.

Cite this article as: Chettiyar VV. Performance Analysis of Emotion Classification Using Multimodal Fusion Technique. J. Comput. Sci. Intell. Technol. 2021; 2(1): 14-20. CJCSIT, MNAA PUB WORLD, 2021. 